\title{
The Classical Limit for Quantum Dynamical Semigroups
}

\author{
E. B. Davies \\ St. John's College, Oxford OX1 3JP, England
}

\begin{abstract}
We describe a class of single-particle quantum-mechanical dynamical semigroups which, in the classical limit, give rise to Markov semigroups on phase space.
\end{abstract}

\section{§1. Introduction}

The close connection between quantum-mechanical dynamical semigroups and Markov semigroups has been considerably clarified recently. Both are particular cases of an abstract theory of stochastic processes [1,2] and the latter can also arise from the former by restricting to a special class of states called quasiclassical or coherent states $[3,11]$. As a new aspect of the connection we show that one obtains Markov semigroups by taking the classical limit of certain dynamical semigroups in a suitable manner. The dynamical semigroups we start with are of the type which arise in the weak or singular coupling limit of a quantum-mechanical particle interacting with an infinite free reservoir $[4,5,8,9,12$, $13,15]$, but we do not pursue this here.

We take the evolution of an open system to be described by a strongly continuous one-parameter "dynamical" semigroup

$$
\left.T_{\lambda}(t)=\exp \left\{\lambda^{-2} Z+K\right) t\right\}
$$

on a Banach space $V$, called the state space. The unbounded operator $Z$ is the generator of a strongly continuous one-parameter group of isometries $e^{Z t}$ on $V$ which determines the free evolution. The bounded operator $K$ describes a perturbation of a "stochastic" type due to the influence of the external world. For reasons given in the references above we examine the asymptotic form of the evolution in the (weak or singular) coupling limit $\lambda \rightarrow 0$, where $\lambda$ is real. In typical cases the effect of $K$ integrated over all time is not finite, so the formalism of scattering theory is not appropriate. Moreover $T_{\lambda}(t)$ is generally a contraction only for $t \geqq 0$, so we restrict attention to such times $t$ from now on. 
The quantum-mechanical applications arise by choosing $V$ to be the space $\mathscr{T}_{s}(\mathscr{H})$ of all self-adjoint trace class operators on a Hilbert space $\mathscr{H}$, with the trace norm. $V$ is partially ordered and the dynamical semigroups of physical interest are positivity-preserving and trace-preserving for all $t \geqq 0$. For simplicity of presentation and generality we develop the theory at the abstract Banach space level, and only return to the quantum-mechanical applications in Section 4.

\section{§ 2. Evolution in the Interaction Picture}

It has been shown in $[5,7]$ that if $V$ is finite dimensional, there exists an operator $K^{\natural} \in \mathscr{L}(V)$, the space of bounded operators on $V$, such that

$$
\lim _{\lambda \rightarrow 0} e^{-\lambda^{-2} Z t} T_{\lambda}(t)=\exp \left\{K^{\natural} t\right\} .
$$

Such a result is also sometimes possible when $V$ is infinite-dimensional.

Theorem 2.1. Suppose that

$$
\lim _{a \rightarrow \infty} a^{-1} \int_{0}^{a} e^{-Z s} K e^{Z s} d s=K^{\natural}
$$

in the strong operator topology. Then

$$
\lim _{\lambda \rightarrow 0} e^{-\lambda^{-2} Z t} T_{\lambda}(t) f=\exp \left\{K^{\natural} t\right) f
$$

uniformly for $t$ in any compact interval, for all $f \in V$.

Proof. This is Theorem 1.4 of [5] except for a slight change in the proof that $\mathscr{H}_{\lambda} \rightarrow \mathscr{H}$ in the strong operator topology.

We say that $Z$ has pure point spectrum if there are $\alpha_{n} \in \mathbb{R}$ and $f_{n} \in V$ such that

$$
Z f_{n}=i \alpha_{n} f_{n}
$$

and the linear span of the $f_{n}$ is dense in $V$.

Theorem 2.2. If $Z$ has pure point spectrum then the limit of Equation (2.1) does exist in the strong operator topology.

Proof. We first show that for every $\alpha \in \mathbb{R}, Z$ has a "spectral projection" $P_{\alpha}$. We define

$$
P_{\alpha}^{s}=s^{-1} \int_{0}^{s} e^{-Z x} e^{i \alpha x} d x
$$

so that $\left\|P_{\alpha}\right\| \leqq 1$ and

$$
\begin{aligned}
\lim _{s \rightarrow \infty} P_{\alpha}^{s} f_{n} & =\lim _{s \rightarrow \infty} s^{-1} \int_{0}^{s} e^{i\left(\alpha-\alpha_{n}\right) x} d x f_{n} \\
& =\delta\left(\alpha, \alpha_{n}\right) \mathrm{f}_{n} .
\end{aligned}
$$


Since lin $\left\{f_{n}\right\}$ is dense in $V, P_{\alpha}^{s}$ converges strongly as $s \rightarrow \infty$ to an operator $P_{\alpha}$ of norm $\leqq 1$ such that

$$
P_{\alpha} f_{n}=\delta\left(\alpha, \alpha_{n}\right) f_{n} .
$$

It is clear that $P_{\alpha}$ is a projection.

If

$$
K_{a}=a^{-1} \int_{0}^{a} e^{-Z s} K e^{Z s} d s
$$

then $\left\|K_{a}\right\| \leqq\|K\|$, so to prove strong convergence of $K_{a}$ as $a \rightarrow \infty$ it is sufficient to prove it on a dense set. This is a consequence of

$$
\lim _{a \rightarrow \infty} K_{a} f_{n}=\lim _{a \rightarrow \infty} a^{-1} \int_{0}^{a} e^{-Z s} e^{i \alpha_{n} s}\left(K f_{n}\right) d s=P_{\alpha_{n}} K f_{n} .
$$

In order to state the next result we define $K$ to be $Z$-local if

$$
\lim _{t \rightarrow \infty}\left\|K e^{Z t} f\right\|=0
$$

for all $f \in V$. It is easy to show that if $V$ is a Hilbert space, $Z$ is skew-adjoint with absolutely continuous spectrum, and $K$ is compact, then $K$ is $Z$-local.

Theorem 2.3. If $K_{1}$ is $Z$-local, $K_{2}$ commutes with $Z$ and $K=K_{1}+K_{2}$, then $K^{\natural}$ exists and equals $K_{2}$.

Proof. It is an immediate consequence of the definition that $K_{1}^{\natural}=0$ and $K_{2}^{\natural}=K_{2}$. Example 2.4. If $\mathscr{H}$ is a Hilbert space, $V=\mathscr{T}_{s}(\mathscr{H}), H$ is a self-adjoint operator on $\mathscr{H}$ and

$$
e^{Z t}(\varrho)=e^{-i H t} \varrho e^{i H t}
$$

then $e^{Z t}$ is a strongly continuous one-parameter group of isometries on $V$. If $\varrho_{0} \in V$ and

$$
K \varrho=\varrho_{0} \operatorname{tr}[\varrho]
$$

then $K$ is a bounded operator on $V$ for which $K^{\natural}$ does not generally exist. The possibility of this example depends on the fact that 0 is not in the point spectrum of $Z$ but is in the point spectrum of $Z^{*}$.

A different type of result concerning the asymptotic form of $T_{\lambda}(t)$ in the limit $\lambda \rightarrow 0$ is now treated. We define

$$
P_{0}=\lim _{a \rightarrow \infty} a^{-1} \int_{0}^{a} e^{-Z s} d s
$$

if this limit exists in the strong operator topology. The existence of the limit if $e^{-Z t}$ is a unitary group on the Hilbert space $V$ may be established by spectral theory. $P_{0}$ does not exist, however, in Example 2.4.

Lemma 2.5. If $P_{0}$ exists, it is a projection of norm one with range

$$
V_{0}=\{f \in V: Z f=0\} .
$$


Proof. If $f \in V_{0}$ then $P_{0} f=f$ and $f$ lies in the range of $P_{0}$. Conversely if $f \in V$

$$
\begin{aligned}
& \left\|e^{-Z t} a^{-1} \int_{0}^{a} e^{-Z s} f d s-a^{-1} \int_{0}^{a} e^{-Z s} f d s\right\| \\
& \leqq 2 t a^{-1} \rightarrow 0
\end{aligned}
$$

as $a \rightarrow \infty$, so

$$
e^{-Z t} P_{0} f=P_{0} f
$$

for all $t \in \mathbb{R}$. Therefore $f \in V_{0}$.

The following theorem is similar to one in [14].

Theorem 2.6. If $P_{0}$ exists and $K_{0}$ is the restriction of $P_{0} K$ to $V_{0}$ then

$$
\lim _{\lambda \rightarrow 0} T_{\lambda}(t) f=e^{K_{0} t} f
$$

uniformly for $t$ in any finite interval, and for all $f \in V_{0}$.

Proof. Given $a>0$ we denote by $\mathscr{W}$ the Banach space of continuous $V$-valued functions on $[0, a]$ and by $\mathscr{W}_{0}$ the subspace of functions with values in $V_{0}$. We first establish that if $g \in \mathscr{W}$ then

$$
\int_{0}^{t} e^{-Z \lambda-2 s} g(s) d s
$$

converges uniformly as $\lambda \rightarrow 0$. By density it is sufficient to prove this when $g$ is continuously differentiable. In this case if

$$
A(a)=a^{-1} \int_{0}^{a} e^{-Z s} d s
$$

then

$$
\begin{aligned}
\int_{0}^{t} e^{-Z \lambda-2 s} g(s) d s= & t A\left(\lambda^{-2} t\right) g(t) \\
& -\int_{0}^{t} s A\left(\lambda^{-2} s\right) g^{\prime}(s) d s
\end{aligned}
$$

which converges uniformly as $\lambda \rightarrow 0$ to

$$
\begin{aligned}
t P_{0} g(t) & -\int_{0}^{t} s P_{0} g^{\prime}(s) d s \\
& =\int_{0}^{t} P_{0} g(s) d s .
\end{aligned}
$$

Given $f(0) \in V_{0}$ we define

$$
f_{\lambda}(t)=\exp \left(-Z \lambda^{-2} t\right) T_{\lambda}(t) f(0)
$$

so that $f_{\lambda} \in \mathscr{W}$ and as in [5]

$$
f_{\lambda}=f(0)+\mathscr{H}_{\lambda} f_{\lambda}
$$


where $\mathscr{H}_{\lambda}: \mathscr{W} \rightarrow \mathscr{W}$ is defined by

$$
\left(\mathscr{H}_{\lambda} g\right)(t)=\int_{0}^{t} e^{-Z \lambda-2 s} K e^{Z \lambda-2 s} g(s) d s .
$$

If $g \in \mathscr{W}_{0}$ then the above argument shows that $\mathscr{H}_{\lambda} g$ converges uniformly as $\lambda \rightarrow 0$ to $\mathscr{H} g$ where $\mathscr{H}: \mathscr{W} \rightarrow \mathscr{W}$ is defined by

$$
(\mathscr{H} g)(t)=\int_{0}^{t} K_{0} g(s) d s .
$$

Thus $\mathscr{H}_{\lambda}$ converges strongly to $\mathscr{H}$ on the subspace $\mathscr{W}_{0}$, which is invariant for $\mathscr{H}$. It follows by induction that $\mathscr{H}_{\lambda}^{n}$ converges strongly to $\mathscr{H}^{n}$ on $\mathscr{W}_{0}$ and hence that

$$
f_{\lambda}=f(0)+\mathscr{H}_{\lambda} f(0)+\mathscr{H}_{\lambda}^{2} f(0)+\ldots
$$

converges in norm to

$$
f=f(0)+\mathscr{H} f(0)+\mathscr{H}^{2} f(0)+\ldots
$$

as $\lambda \rightarrow 0$, using the estimate

$$
\left\|\mathscr{H}_{\lambda}^{n}\right\| \leqq a^{n}\|K\|^{n} / n !
$$

But $f \in \mathscr{W}_{0}$ is the solution of

$$
f(t)=f(0)+\int_{0}^{t} K_{0} f(s) d s
$$

so $f(t)=\exp \left(K_{0} t\right) f(0)$. It follows that

$$
\begin{aligned}
\lim _{\lambda \rightarrow 0} & \sup _{0 \leqq t \leqq a}\left\|T_{\lambda}(t) f(0)-e^{K_{0} t} f(0)\right\| \\
& =\lim _{\lambda \rightarrow 0} \sup _{0 \leqq t \leqq a}\left\|e^{-Z \lambda^{-2} t} T_{\lambda}(t) f(0)-e^{-Z \lambda-2 t} e^{K_{0} t} f(0)\right\| \\
& =\lim _{\lambda \rightarrow 0} \sup _{0 \leqq t \leqq a}\left\|e^{-Z \lambda^{-2} t} T_{\lambda}(t) f(0)-e^{K_{0} t} f(0)\right\| \\
& =0 .
\end{aligned}
$$

Example 2.7. If $V$ is the space of $n \times n$ matrices, $H$ is a diagonal self-adjoint matrix with distinct eigenvalues, and

$$
e^{Z t}(\varrho)=e^{-i H t} \varrho e^{i H t}
$$

then $P_{0}$ exists and $V_{0}$ is the space of diagonal matrices.

Example 2.8. If $\mathscr{H}=L^{2}(\mathbb{R})$ and $V=\mathscr{T}_{s}(\mathscr{H})$ and $(H \psi)(k)=\frac{1}{2} k^{2} \psi(k)$ for all $\psi \in \mathscr{H}$, then $P_{0}$ does not exist. There is however an operator

$$
P_{0}: \mathscr{T}_{s}(\mathscr{H}) \rightarrow L^{1}(\mathbb{R})
$$

which plays the same role. If $\varrho \in V$ has integral kernel $\varrho\left(k, k^{\prime}\right)$ then

$$
\left(P_{0} \varrho\right)(k)=\varrho(k ; k) \text {. }
$$


Alternatively if

$$
\varrho=\sum_{n=1}^{\infty} \lambda_{n}\left|\psi_{n}\right\rangle\left\langle\psi_{n}\right|
$$

then

$$
\left(P_{0} \varrho\right)(k)=\sum_{n=1}^{\infty} \lambda_{n}\left|\psi_{n}(k)\right|^{2} .
$$

It may easily be verified that $P_{0}$ is positive and linear and that

$$
\int_{R}\left(P_{0} \varrho\right)(k) d k=\operatorname{tr}[\varrho]
$$

for all $\varrho \in V$.

Theorem 2.9. Let $P_{0}$ be a bounded operator from the Banach space $V$ into the Banach space $V_{0}$. Let $e^{C t}$ be a strongly continuous one-parameter contraction semigroup on $V_{0}$, let $e^{D t}$ be a strongly continuous one-parameter contraction semigroup on $V$ and let

$$
C P_{0} f=P_{0} D f
$$

for all $f$ in some core $\mathscr{D}$ of $D$. Then

$$
e^{C t} P_{0} f=P_{0} e^{D t} f
$$

for all $f \in V$ and all $t \geqq 0$.

Proof. $C$ and $D$ are closed operators and if $f \in \operatorname{dom}(D)$ then there is a sequence $f_{n} \in \mathscr{D}$ such that $f_{n} \rightarrow f$ and $D f_{n} \rightarrow D f$. Then $P_{0} f_{n} \rightarrow P_{0} f$ and $C\left(P_{0} f_{n}\right) \rightarrow P_{0} D f$ by Equation (2.14). Therefore $P_{0} f \in \operatorname{dom}(C)$ and

$$
C P_{0} f=P_{0} D f \text {. }
$$

If $f \in \operatorname{dom}(D)$ then $e^{D s} f \in \operatorname{dom}(D)$ for all $s \geqq 0$ and

$$
\begin{aligned}
& \frac{d}{d s} e^{C(t-s)} P_{0} e^{D s} f \\
& \quad=e^{C(t-s)}\left(-C P_{0}+P_{0} D\right) e^{D s} f \\
& \quad=0
\end{aligned}
$$

so

$$
e^{C t} P_{0} f=P_{0} e^{D t} f .
$$

The same holds for all $f \in V$ by density.

The above theorem will be used in Section 4 to relate a quantum dynamical semigroup to a Markov semigroup on momentum space.

\section{§ 3. Asymptotic Limits between Two Spaces}

When one tries to relate a quantum dynamical semigroup to a Markov semigroup on phase space, difficulties arise immediately because of the non-existence of a canonical phase space distribution for an arbitrary state. One has therefore to 
allow the projection between the spaces $V$ and $V_{0}$ to depend on $\lambda$, and to take the classical limit at the same time as the limit $\lambda \rightarrow 0$. We write down in this section only the abstract part of the theory, the applications being in Section 4.

Throughout this section we suppose that $T_{\lambda}(t)=e^{Z_{\lambda t}}$ is a strongly continuous one-parameter contraction semigroup on the Banach space $V$ for all small enough $\lambda>0$. We suppose that $P_{\lambda}: V \rightarrow V_{0}$ are operators of norm one into the Banach space $V_{0}$ for all small enough $\lambda>0$. We also suppose that $T_{0}(t)$ is a (not necessarily continuous) one-parameter contraction semigroup on $V_{0}$, with infinitesimal generator $Z_{0}$ which need not be densely defined, but is always closed [6].

Theorem 3.1. Let $\mathscr{D}$ be a core of all $Z_{\lambda}$ and let $P_{\lambda} \mathscr{D} \cong \operatorname{dom}\left(Z_{0}\right)$ for all $\lambda$. Suppose that if $f \in \mathscr{D}$ then

$$
\left\|Z_{0} P_{\lambda} f-P_{\lambda} Z_{\lambda} f\right\| \leqq K_{\lambda}\|f\|+L_{\lambda}\left\|Z_{\lambda} f\right\|
$$

where $K_{\lambda}$ and $L_{\lambda}$ are independent of $f$. Suppose also that if $f \in \mathscr{D}$ then

$$
\lim _{\lambda \rightarrow 0}\left\{K_{\lambda}\|f\|+L_{\lambda}\left\|Z_{\lambda} f\right\|\right\}=0 .
$$

Then

$$
\lim _{\lambda \rightarrow 0}\left\|T_{0}(t) P_{\lambda} f-P_{\lambda} T_{\lambda}(t) f\right\|=0
$$

for all $f \in V$, uniformly for $t$ in any finite interval.

Proof. $\operatorname{dom}\left(Z_{\lambda}\right)$ is a Banach space for the norm

$$
\|f\|_{\lambda}=K_{\lambda}\|f\|+L_{\lambda}\left\|Z_{\lambda} f\right\|
$$

and $\left(Z_{0} P_{\lambda}-P Z_{\lambda}\right)$ can be extended from $\mathscr{D}$ to a contraction $A_{\lambda}: \operatorname{dom}\left(Z_{\lambda}\right) \rightarrow V_{0}$. If $f \in \operatorname{dom}\left(Z_{\lambda}\right)$ then there exist $f_{n} \in \mathscr{D}$ such that $f_{n} \rightarrow f$ and $Z_{\lambda} f_{n} \rightarrow Z_{\lambda} f$. Therefore $\left\|f_{n}-f\right\|_{\lambda \rightarrow 0}$ and

$$
\begin{aligned}
Z_{0}\left(P_{\lambda} f_{n}\right)= & P_{\lambda} Z_{\lambda} f_{n}+A_{\lambda} f_{n} \\
& \rightarrow P_{\lambda} Z_{\lambda} f+A_{\lambda} f .
\end{aligned}
$$

It follows that $P_{\lambda} f \in \operatorname{dom} Z_{0}$ and

$$
Z_{0} P_{\lambda} f-P_{\lambda} Z_{\lambda} f=A_{\lambda} f
$$

for all $f \in \operatorname{dom}\left(Z_{\lambda}\right)$. The inequality (3.1) therefore holds for all $f \in \operatorname{dom}\left(Z_{\lambda}\right)$, which is invariant under $T_{\lambda}(t)$. For such $f$

$$
\begin{aligned}
& \left\|\frac{d}{d s} e^{Z_{0}(t-s)} P_{\lambda} e^{Z_{\lambda s}} f\right\| \\
& \quad=\left\|e^{Z_{0}(t-s)}\left(-Z_{0} P_{\lambda}+P_{\lambda} Z_{\lambda}\right) e^{Z_{\lambda s}} f\right\| \\
& \quad \leqq K_{\lambda}\left\|e^{Z_{\lambda s}} f\right\|+L_{\lambda}\left\|Z_{\lambda} e^{Z_{\lambda s}} f\right\| \\
& \quad \leqq K_{\lambda}\|f\|+L_{\lambda}\left\|Z_{\lambda} f\right\| .
\end{aligned}
$$


Therefore if $f \in \mathscr{D}$

$$
\left\|e^{Z_{0} t} P_{\lambda} f-P_{\lambda} e^{Z_{\lambda} t} f\right\| \leqq t\left\{K_{\lambda}\|f\|+L_{\lambda}\left\|Z_{\lambda} f\right\|\right\}
$$

which converges to zero as $\lambda \rightarrow 0$. The same holds for all $f \in V$ by density.

We say that $T_{0}(t)$ is a dual semigroup if $V_{0}$ is the Banach dual of a space $W$ and $T_{0}(t)$ is the adjoint of a strongly continuous one-parameter contraction semigroup on $W$.

Theorem 3.2. Suppose that $P_{\lambda}: V \rightarrow V_{0}$ are contractions for $\lambda \geqq 0$ and that for all $f \in V$

$$
\lim _{\lambda \rightarrow 0} P_{\lambda} f=P_{0} f
$$

in the weak* topology of $V_{0}$. If $T_{0}(t)$ is a dual semigroup on $V_{0}$ and the conditions of Theorem 3.1 are satisfied then

$$
\lim _{\lambda \rightarrow 0} P_{\lambda} T_{\lambda}(t) f=T_{0}(t) P_{0} f
$$

in the weak* topology of $V_{0}$, for all $f \in V$ and $t \geqq 0$.

Proof. We combine Theorem 3.1 with the observation that since $T_{0}(t)$ is weak* continuous

$$
\lim _{\lambda \rightarrow 0} T_{0}(t) P_{\lambda} f=T_{0}(t) P_{0} f
$$

in the weak* topology of $V_{0}$ for all $f \in V$ and $t \geqq 0$.

\section{§ 4. Markov Semigroups on Phase Space}

We consider a certain quantum dynamical semigroup on the state space $V=\mathscr{T}_{s}(\mathscr{H})$ of a single spinless particle in one dimension, so that $\mathscr{H}=L^{2}(\mathbb{R})$. The free Hamiltonian is given in the momentum space representation by

$$
(H \psi)(k)=\frac{1}{2} k^{2} \psi(k)
$$

on the usual domain, and

$$
e^{Z t}(\varrho)=e^{-i H t} \varrho e^{i H t}
$$

defines a strongly continuous one-parameter group of isometries on $V$ whose infinitesimal generator $Z$ is given formally by

$$
Z(\varrho)=-i[H, \varrho]
$$

or by

$$
(Z \varrho)(h, k)=\frac{i}{2}\left(k^{2}-h^{2}\right) \varrho(h, k)
$$

in terms of the momentum space kernel of $\varrho$. The domain $\mathscr{D}$ of all $\varrho \in V$ whose integral kernels in momentum space are continuously differentiable and of compact support is dense and invariant under $e^{Z t}$ and therefore is a core for $Z$. 
The Weyl operators $W(k, x)$ are defined on $L^{2}(\mathbb{R})$ by

$$
\{W(k, x) \psi\}(h)=\exp [i x k / 2-i x h] \psi(h-k)
$$

and satisfy the relation

$$
W(k, x) W\left(k^{\prime}, x^{\prime}\right)=W\left(k+k^{\prime}, x+x^{\prime}\right) \exp \left[i\left(k x^{\prime}-k^{\prime} x\right) / 2\right] .
$$

We define a positive definite measure $\sigma$ on $\mathbb{R}^{3}$ as a complex measure with a decomposition

$$
\sigma(d a, d b, d h)=\sum_{n=1}^{\infty} \mu_{n}(d a) \overline{\mu_{n}(d b)} v_{n}(d h)
$$

where $\mu_{n}$ are complex measures, $v_{n}$ is a positive measure and

$$
\|\sigma\| \equiv \sum_{n=1}^{\infty}\left\|\mu_{n}\right\|^{2}\left\|v_{n}\right\|<\infty .
$$

A larger class of measures $\sigma$ can no doubt be allowed in the following theory. Theorem 4.1. If $\sigma$ is a positive definite measure on $\mathbb{R}^{3}$ define $B: V \rightarrow V$ by

$$
B(\varrho)=\int_{\mathbb{R}^{3}} W(h, b)^{*} \varrho W(h, a) \sigma(d a, d b, d h)
$$

and $R \in \mathscr{L}(\mathscr{H})$ by

$$
R=\int_{\mathbb{R}^{3}} W(h, a) W(h, b)^{*} \sigma(d a, d b, d h) .
$$

Then the closure of the operator $Z_{\lambda}$ defined on $\mathscr{D}$ by

$$
Z_{\lambda}(\varrho)=\lambda^{-2} Z(\varrho)+B(\varrho)-\frac{1}{2}(R \varrho+\varrho R)
$$

is the infinitesimal generator of a strongly continuous one parameter contraction semigroup $T_{\lambda}(t)$ on $V$. Moreover $T_{\lambda}(t)$ is positivity and trace preserving for all $t \geqq 0$. Proof. If $B_{n h}$ is the bounded operator

$$
B_{n h}=\int_{\mathbb{R}} W(h, a) \mu_{n}(d a)
$$

then

$$
B(\varrho)=\sum_{n=1}^{\infty} B_{n h}^{*} \varrho B_{n h} v_{n}(d h)
$$

so $B$ is a bounded and positivity preserving operator on $V$. The operator $R$ satisfies

$$
\operatorname{tr}[R \varrho]=\operatorname{tr}[B(\varrho)]
$$

for all $\varrho \in V$. The derivation of the properties of $T_{\lambda}(t)$ may now be found in $[1,2]$.

The dynamical semigroup $T_{\lambda}(t)$ is of the type which has been obtained in a weak or singular coupling limit $[4,8,13,15]$ of a particle interacting with an infinite reservoir. We can relate it to a Markov semigroup on momentum space with little difficulty. Let $P_{0}: \mathscr{T}_{s}(\mathscr{H}) \rightarrow L^{1}(\mathbb{R})$ be the projection of Example 2.8. 
Theorem 4.2. Let $T(t)$ be the norm continuous Markov semigroup on $L^{1}(\mathbb{R})$ with infinitesimal generator given by

$$
\begin{aligned}
\left\{\left(B_{0}-R_{0}\right) f\right\}(k)= & \sum_{n=1}^{\infty} \int_{\mathbb{R}}\left|\hat{\mu}_{n}(k+h / 2)\right|^{2} f(k+h) v_{n}(d h) \\
& -\sum_{n=1}^{\infty} \int_{\mathbb{R}}\left|\hat{\mu}_{n}(k-h / 2)\right|^{2} v_{n}(d h) f(k)
\end{aligned}
$$

where $\hat{\mu}_{n}$ is the Fourier transform of $\mu_{n}$. Then

$$
T(t) P_{0} \varrho=P_{0} T_{\lambda}(t) \varrho
$$

for all $\varrho \in V, t \geqq 0$ and $\lambda>0$.

Proof. By Equation (2.10) it is clear that $P_{0} Z \varrho=0$ for all $\varrho \in \mathscr{D}$. The integral kernel of $B(\varrho)$ is

$$
\begin{aligned}
& (B \varrho)\left(k, k^{\prime}\right)=\sum_{n=1}^{\infty} \int_{\mathbb{R}^{3}} \exp [i b h / 2+i b k] \varrho\left(k+h, k^{\prime}+h\right) . \\
& \exp \left[-i a h / 2-i a k^{\prime}\right] \mu_{n}(d a) \overline{\mu_{n}(d b)} v_{n}(d h) \\
& \quad=\sum_{n=1}^{\infty} \int_{\mathbb{R}} \hat{\mu}_{n}\left(k^{\prime}+h / 2\right) \overline{\hat{\mu}_{n}(k+h / 2)} \varrho\left(k+h, k^{\prime}+h\right) v_{n}(d h) .
\end{aligned}
$$

Therefore

$$
\begin{aligned}
\left(P_{0} B \varrho\right)(k) & =\sum_{n=1}^{\infty} \int_{\mathbb{R}}\left|\hat{\mu}_{n}(k+h / 2)\right|^{2}\left(P_{0} \varrho\right)(k+h) v_{n}(d h) \\
& =\left(B_{0} P_{0} \varrho\right)(k) .
\end{aligned}
$$

Similarly

$$
\begin{aligned}
& (R \varrho+\varrho R)\left(k, k^{\prime}\right)=\sum_{n=1}^{\infty} \int_{\mathbb{R}^{3}} \exp [i(h a-h b) / 2] \\
& \{W(0, a-b) \varrho\}\left(k, k^{\prime}\right) \mu_{n}(d a) \overline{\mu_{n}(d b)} v_{n}(d h)+\text { conj. } \\
& \quad=\sum_{n=1}^{\infty} \int_{\mathbb{R}^{3}} \exp [i(a-b)(h / 2-k)] \varrho\left(k, k^{\prime}\right) \mu_{n}(d a) \overline{\mu_{n}(d b)} v_{n}(d h)+\text { conj. } \\
& \quad=\sum_{n=1}^{\infty} \int_{\mathbb{R}}\left|\hat{\mu}_{n}(k-h / 2)\right|^{2} \varrho\left(k, k^{\prime}\right) v_{n}(d h)+\text { conj. }
\end{aligned}
$$

Therefore

$$
\begin{aligned}
& \left\{P_{0}(R \varrho+\varrho R)\right\}(k) \\
& \quad=2 \sum_{n=1}^{\infty} \int_{\mathbb{R}}\left|\hat{\mu}_{n}(k-h / 2)\right|^{2} v_{n}(d h)\left(P_{0} \varrho\right)(k) \\
& \quad=\left(2 R_{0} P_{0} \varrho\right)(k) .
\end{aligned}
$$

The proof is completed by an application of Theorem 2.9. 
We now define the Banach space $V_{0}$ to be the space of all finite complex measures on phase space $\mathbb{R}^{2}$, with the usual norm. $V_{0}$ is the Banach dual space of the space $C_{0}\left(\mathbb{R}^{2}\right)$ of all continuous functions on $\mathbb{R}^{2}$ which vanish at infinity, and contains $L^{1}\left(\mathbb{R}^{2}\right)$ as a weak ${ }^{*}$ dense subspace.

We let $e^{Z_{* t}}$ be the strongly continuous one-parameter contraction semigroup on $C_{0}\left(\mathbb{R}^{2}\right)$ with infinitesimal generator

$$
\begin{aligned}
\left(Z_{*} f\right)(k, x)= & k \frac{\partial f}{\partial x} \\
& +\sum_{n=1}^{\infty} \int_{\mathbb{R}}\left|\hat{\mu}_{n}(k-h / 2)\right|^{2} f(k-h, x) v_{n}(d h) \\
& -\sum_{n=1}^{\infty} \int_{\mathbb{R}}\left|\hat{\mu}_{n}(k-h / 2)\right|^{2} v_{n}(d h) f(k, x) .
\end{aligned}
$$

The dual semigroup $T_{0}(t)$ on $V_{0}$ is a Markov semigroup; in other words if $\mu$ is a probability measure on $\mathbb{R}^{2}$ then so is $T_{0}(t) \mu$ for all $t \geqq 0$. The semigroup $T_{0}(t)$ leaves $L^{1}\left(\mathbb{R}^{2}\right)$ invariant and on this subspace is strongly continuous with infinitesimal generator $Z_{0}$ given by

$$
\begin{aligned}
\left(Z_{0} f\right)(k, x)= & -k \frac{\partial f}{\partial x} \\
& +\sum_{n=1}^{\infty} \int_{\mathbb{R}}\left|\hat{\mu}_{n}(k+h / 2)\right|^{2} f(k+h, x) v_{n}(d h) \\
& -\sum_{n=1}^{\infty} \int_{\mathbb{R}}\left|\hat{\mu}_{n}(k-h / 2)\right|^{2} v_{n}(d h) f(k, x) \\
= & \left(C_{0} f+B_{0} f-R_{0} f\right)(k, x)
\end{aligned}
$$

say. Note that

$$
\left(e^{C_{0} t} f\right)(k, x)=f(k, x-k t)
$$

describes free classical motion on phase space and that $B_{0}$ and $R_{0}$ are the phase space versions of the corresponding operators of Theorem 4.2. Therefore $T_{0}(t)$ physically describes free motion of a classical particle subject to random impulses.

It is somewhat difficult to associate the Markov semigroup $T_{0}(t)$ with the quantum dynamical semigroup $T_{\lambda}(t)$ because a state $\varrho \in V$ does not have a canonical phase space distribution. As $\lambda \rightarrow 0$ the following maps define a scaling of the states similar to one used in [10].

Lemma 4.3. If $\varphi, \psi \in \mathscr{H}$ the formula

$$
\left(P_{\varphi, \psi}^{\lambda} \varrho\right)(k, x)=\frac{1}{2 \pi \lambda^{2}}\left\langle\varrho W\left(k, \lambda^{-2} x\right) \varphi, W\left(k, \lambda^{-2} x\right) \psi\right\rangle
$$

defines a bounded linear map

$$
P_{\varphi \psi}^{\lambda}: \mathscr{T}_{s}(\mathscr{H}) \rightarrow L^{1}\left(\mathbb{R}^{2}\right)
$$


with

$$
\left\|P_{\varphi, \psi}^{\lambda}\right\| \leqq\|\varphi\|\|\psi\|
$$

If $\varphi=\psi$ is a vector of norm one then $P_{\varphi}^{\lambda}$ is positivity preserving and

$$
\int_{\mathbb{R}^{2}}\left(P_{\varphi, \varphi}^{\lambda} \varrho\right)(k, x) d k d x=\operatorname{tr}[\varrho]
$$

for all $\varrho \in V$.

Proof. By a scale change we may assume that $\lambda=1$, and by the spectral decomposition of $\varrho$ we may assume that it is a pure state $\varrho=|\xi\rangle\langle\xi|$. It is therefore enough to prove that

$$
\frac{1}{2 \pi} \int_{\mathbb{R}^{2}}|\langle W(k, x) \varphi, \xi\rangle|^{2} d k d x=\|\varphi\|^{2}\|\xi\|^{2}
$$

for all $\varphi, \xi \in \mathscr{H}$. But

$$
\begin{gathered}
(2 \pi)^{-\frac{1}{2}}\langle W(k, x) \varphi, \xi\rangle \exp [-i x k / 2] \\
\quad=(2 \pi)^{-\frac{1}{2}} \int_{\mathbb{R}} e^{-i x h} \varphi(h-k) \overline{\xi(h)} d h .
\end{gathered}
$$

Therefore by the Plancherel theorem

$$
\begin{aligned}
& \frac{1}{2 \pi} \int_{\mathbb{R}^{2}}|\langle W(k, x) \varphi, \xi\rangle|^{2} d x d k \\
& \quad=\int_{\mathbb{R}^{2}}|\varphi(h-k) \overline{\xi(h)}|^{2} d h d k \\
& \quad=\int_{\mathbb{R}^{2}}|\varphi(k)|^{2}|\xi(h)|^{2} d h d k=\|\varphi\|^{2}\|\xi\|^{2}
\end{aligned}
$$

as required.

In order to apply Theorem 3.2 we need the following result.

Theorem 4.4. Let $\varphi \in L^{2}(\mathbb{R})$ be a unit vector in Schwartz space and define the unit vector $\varphi_{\lambda}$ by

$$
\varphi_{\lambda}(k)=\lambda^{-\beta / 2} \varphi\left(\lambda^{-\beta} k\right)
$$

where $1<\beta<2$. If $P_{\lambda} \equiv P_{\varphi_{\lambda}, \varphi_{\lambda}}^{\lambda}$ for all $\lambda>0$ and $P_{0}: V \rightarrow V_{0}$ is defined by

$$
\left(P_{0} \varrho\right)(d k, d x)=\varrho(k, k) d k \delta_{0}(d x)
$$

then

$$
\lim _{\lambda \rightarrow 0} P_{\lambda} \varrho=P_{0} \varrho
$$

in the weak* topology of $V_{0}$ for all $\varrho \in V$.

Proof. By density arguments it is sufficient to prove that

$$
\lim _{\lambda \rightarrow 0} \int_{\mathbb{R}^{2}}\left(P_{\lambda} \varrho\right)(k, x) f(k, x) d k d x=\int_{\mathbb{R}} \varrho(k, k) f(k, 0) d k
$$


whenever $\varrho \in \mathscr{D}$ and $f$ is continuous and of compact support. For such $\varrho$ and $f$

$$
\lim _{\lambda \rightarrow 0} \int_{\mathbb{R}^{2}} \varrho(k, k) \lambda^{\beta-2}\left|\hat{\varphi}\left(\lambda^{\beta-2} x\right)\right|^{2} f(k, x) d k d x=\int_{\mathbb{R}} \varrho(k, k) f(k, 0) d k
$$

where $\hat{\varphi}$ is the Fourier transform of $\varphi$. Moreover

$$
\begin{aligned}
& \mid \int_{\mathbb{R}^{2}}\left(P_{\lambda} \varrho\right)(k, x) f(k, x) d k d x \\
& \quad-\int_{\mathbb{R}^{2}} \varrho(k, k) \lambda^{\beta-2}\left|\hat{\varphi}\left(\lambda^{\beta-2} x\right)\right|^{2} f(k, x) d k d x \mid \\
& \quad \leqq\left.\|f\|_{1} \sup _{k x}\left|\left(P_{\lambda} \varrho\right)(k, x)-\varrho(k, k) \lambda^{\beta-2}\right| \hat{\varphi}\left(\lambda^{\beta-2} x\right)\right|^{2} \mid .
\end{aligned}
$$

Also

$$
\begin{aligned}
\left.\left|\left(P_{\lambda} \varrho\right)(k, x)-\varrho(k, k) \lambda^{\beta-2}\right| \hat{\varphi}\left(\lambda^{\beta-2} x\right)\right|^{2} \mid \\
=\frac{1}{2 \pi \lambda^{2}} \mid \int_{\mathbb{R}^{2}} \varrho\left(h, h^{\prime}\right) e^{-i \lambda^{-2} x h^{\prime}} \varphi_{\lambda}\left(h^{\prime}-k\right) e^{i \lambda^{-2} x h} \overline{\varphi_{\lambda}(h-k)} d h d h^{\prime} \\
\quad-\int_{\mathbb{R}^{2}} \varrho(k, k) e^{-i \lambda^{-2} x\left(h^{\prime}-h\right)} \varphi_{\lambda}\left(h^{\prime}\right) \overline{\varphi_{\lambda}(h)} d h d h^{\prime} \mid \\
\left.=\frac{1}{2 \pi \lambda^{2}} \mid \int_{\mathbb{R}^{2}} \varrho\left(k+h, k+h^{\prime}\right)-\varrho(k, k)\right\} . \\
\quad e^{i \lambda-2 x\left(h-h^{\prime}\right)} \varphi_{\lambda}\left(h^{\prime}\right) \overline{\varphi_{\lambda}(h)} d h d h^{\prime} \mid \\
\quad \frac{1}{2 \pi \lambda^{2}} \int_{\mathbb{R}^{2}} c\left(|h|+\mid h^{\prime}\right)\left|\varphi_{\lambda}\left(h^{\prime}\right)\right|\left|\varphi_{\lambda}(h)\right| d h d h^{\prime} \\
=\frac{c}{2 \pi} \lambda^{2 \beta-2} \int_{\mathbb{R}^{2}}\left(|h|+\left|h^{\prime}\right|\right)\left|\varphi\left(h^{\prime}\right)\right||\varphi(h)| d h d h^{\prime}
\end{aligned}
$$

which converges to zero as $\lambda \rightarrow 0$ uniformly with respect to $k$ and $x$. This completes the proof.

In the following theorem, the main result of this paper, we take $P_{\lambda}$ and $P_{0}$ to be defined as in Theorem 4.4.

Theorem 4.5. If $\varrho \in V$ and $t \geqq 0$ then

$$
\lim _{\lambda \rightarrow 0} P_{\lambda} T_{\lambda}(t) \varrho=T_{0}(t) P_{0} \varrho
$$

in the weak ${ }^{*}$ topology of $V_{0}$.

Proof. By Theorem 3.2 we need only verify that the conditions of Theorem 3.1 are satisfied. We verify the inequality (3.1) for each term of $Z_{\lambda}$ in Equation (4.6) separately, the core $\mathscr{D}$ being the space defined at the beginning of this section.

Lemma 4.6. There is a constant $K_{\lambda}$ such that $K_{\lambda} \rightarrow 0$ as $\lambda \rightarrow 0$ and

$$
\left\|C_{0} P_{\lambda} \varrho-P_{\lambda} \lambda^{-2} Z \varrho\right\|_{1} \leqq K_{\lambda}\|\varrho\|_{\mathrm{tr}}
$$

for all $\varrho \in \mathscr{D}$. 
Proof. If $\varrho \in \mathscr{D}$ then

$$
\begin{aligned}
\left(P_{\lambda} \lambda^{-2} Z \varrho\right)(k, x)-\left(C_{0} P_{\lambda} \varrho\right)(k, x) \\
=\frac{1}{2 \pi \lambda^{2}} \int_{\mathbb{R}^{2}} \frac{i}{2 \lambda^{2}}\left(h^{\prime 2}-h^{2}\right) \varrho\left(h, h^{\prime}\right) e^{-i \lambda-2 x h^{\prime}} \varphi_{\lambda}\left(h^{\prime}-k\right) . \\
\quad \cdot e^{i \lambda-2 x h} \overline{\varphi_{\lambda}(h-k)} d h d h^{\prime} \\
\quad+\frac{1}{2 \pi \lambda^{2}} \int_{\mathbb{R}^{2}}\left(-k i \lambda^{-2} h^{\prime}+k i \lambda^{-2} h\right) \varrho\left(h, h^{\prime}\right) e^{-i \lambda-2 x h^{\prime}} \varphi_{\lambda}\left(h^{\prime}-k\right) \\
=\frac{i}{4 \pi \lambda^{4}} \int_{\mathbb{R}^{2}}\left\{\left(h^{\prime}-k\right)^{2}-(h-k)^{2}\right\} \varrho\left(h, h^{\prime}\right) \\
\quad \cdot e^{-i \lambda^{-2} x h^{\prime}} \varphi_{\lambda}\left(h^{\prime}-k\right) e^{i \lambda-2 x h} \overline{\varphi_{\lambda}(h-k)} d h d h^{\prime} \\
=\frac{i}{2} \lambda^{2 \beta-2}\left\{\left(P_{\psi_{\lambda}, \varphi_{\lambda}}^{\lambda} \varrho\right)(k, x)-\left(P_{\varphi_{\lambda} \cdot \psi \lambda}^{\lambda} \varrho\right)(k, x)\right\}
\end{aligned}
$$

where

$$
\psi_{\lambda}(h)=\lambda^{-5 \beta / 2} h^{2} \varphi\left(\lambda^{-\beta} h\right)
$$

has $L^{2}$-norm independent of $\lambda$. Therefore by Lemma 4.3

$$
\begin{aligned}
& \left\|P_{\lambda} \lambda^{-2} Z \varrho-C_{0} P_{\lambda} \varrho\right\|_{1} \\
& \quad \leqq \lambda^{2 \beta-2}\left\|\psi_{\lambda}\right\|\left\|\varphi_{\lambda}\right\|\left\|_{\varrho}\right\|_{\mathrm{tr}} \\
& \quad=\lambda^{2 \beta-2}\|\psi\|\|\varphi\|\left\|_{\varrho}\right\|_{\mathrm{tr}} .
\end{aligned}
$$

The proof is completed by putting

$$
K_{\lambda}=\lambda^{2 \beta-2}\|\psi\| \cdot\|\varphi\| .
$$

Lemma 4.7. There is a constant $K_{\lambda}$ such that $K_{\lambda} \rightarrow 0$ as $\lambda \rightarrow 0$ and

$$
\left\|B_{0} P_{\lambda} \varrho-P_{\lambda} B \varrho\right\|_{1} \leqq K_{\lambda}\left\|_{\varrho}\right\|_{\mathrm{tr}}
$$

for all $\varrho \in \mathscr{D}$.

Proof. We have to compare

$$
\begin{aligned}
\left(P_{\lambda} B \varrho\right)(k, x)= & \frac{1}{2 \pi \lambda^{2}} \int_{\mathbb{R}^{3}}\left\langle\varrho W(h, a) W\left(k, \lambda^{-2} x\right) \varphi_{\lambda},\right. \\
& \left.W(h, b) W\left(k, \lambda^{-2} x\right) \varphi_{\lambda}\right\rangle \sigma(d a, d b, d h)
\end{aligned}
$$

with

$$
\begin{aligned}
\left(B_{0} P_{\lambda} \varrho\right)(k, x)= & \frac{1}{2 \pi \lambda^{2}} \sum_{n=1}^{\infty} \int_{\mathbb{R}}\left|\hat{\mu}_{n}(k+h / 2)\right|^{2} \\
& \cdot\left\langle\varrho W\left(k+h, \lambda^{-2} x\right) \varphi_{\lambda}, W\left(k+h, \lambda^{-2} x\right) \varphi_{\lambda}\right\rangle v_{n}(d h) .
\end{aligned}
$$


The quantity to estimate is

$$
\begin{aligned}
& \int_{\mathbb{R}^{2}}\left|\left(P_{\lambda} B \varrho\right)(k, x)-\left(B_{0} P_{\lambda} \varrho\right)(k, x)\right| d k d x \\
& \leqq \frac{1}{2 \pi \lambda^{2}} \sum_{n=1}^{\infty} \int_{\mathbb{R}^{5}} \mid\left\langle\varrho W(h, a) W\left(k, \lambda^{-2} x\right) \varphi_{\lambda}, W(h, b) W\left(k, \lambda^{-2} x\right) \varphi_{\lambda}\right\rangle \\
& \quad-\left\langle\varrho W\left(k+h, \lambda^{-2} x\right) \varphi_{\lambda}, W\left(k+h, \lambda^{-2} x\right) \varphi_{\lambda}\right\rangle \\
& \cdot \exp [i(k+h / 2)(b-a)]|| \mu_{n}|(d a)| \mu_{n} \mid(d b) v_{n}(d h) d k d x .
\end{aligned}
$$

By the dominated convergence theorem it is sufficient to show that for each $a, b, h$ the integral with respect to $k, x$ converges to zero with $\lambda$ in a suitable manner.

$$
\begin{aligned}
I_{\lambda}= & \frac{1}{2 \pi} \int_{\mathbb{R}^{2}} \mid\left\langle\varrho W(h, a) W(k, x) \varphi_{\lambda}, W(h, b) W(k, x) \varphi_{\lambda}\right\rangle \\
& -\left\langle\varrho W(k+h, x) \varphi_{\lambda}, W(k+h, x) \varphi_{\lambda}\right\rangle \exp [i(k+h / 2)(b-a)] \mid d k d x \\
= & \frac{1}{2 \pi} \int_{\mathbb{R}^{2}} \mid\left\langle\varrho W(k, x) W(h, a) \varphi_{\lambda}, W(k, x) W(h, b) \varphi_{\lambda}\right\rangle \\
& -\left\langle\varrho W(k, x) W(h, 0) \varphi_{\lambda}, W(k, x) W(h, 0) \varphi_{\lambda}\right\rangle \exp [i h(b-a) / 2] \mid d k d x \\
= & \| P_{W(h, a) \varphi_{\lambda}, W(h . b) \varphi_{\lambda} \varrho-P_{e^{-i n a / 2}}^{\lambda} W(h, 0) \varphi_{\lambda}, e^{-i h b / 2} W(h, 0) \varphi_{\lambda} \varrho \|_{1}}^{\lambda} \\
\leqq & \left\{\left\|W(h, a) \varphi_{\lambda}-e^{-i h a / 2} W(h, 0) \varphi_{\lambda}\right\|\right. \\
& \left.+\left\|W(h, b) \varphi_{\lambda}-e^{-i h b / 2} W(h, 0) \varphi_{\lambda}\right\|\right\}\left\|_{\varrho}\right\|_{\operatorname{tr}}
\end{aligned}
$$

by Lemma 4.3. Therefore

$$
I_{\lambda} \leqq\left\{\left\|W(0, a) \varphi_{\lambda}-\varphi_{\lambda}\right\|+\left\|W(0, b) \varphi_{\lambda}-\varphi_{\lambda}\right\|\right\}\left\|_{\varrho}\right\|_{\mathrm{tr}}
$$

which does indeed converge to zero as $\lambda \rightarrow 0$.

Lemma 4.8. There is a constant $K_{\lambda}$ such that $K_{\lambda} \rightarrow 0$ as $\lambda \rightarrow 0$ and

$$
\left\|R_{0} P_{\lambda}-\frac{1}{2} P_{\lambda}(R \varrho+\varrho R)\right\|_{1} \leqq K_{\lambda}\left\|_{\varrho}\right\|_{\mathrm{tr}}
$$

for all $\varrho \in \mathscr{D}$.

Proof. We have to compare

$$
\begin{aligned}
\left(P_{\lambda}(\varrho R)\right)(k, x)= & \frac{1}{2 \pi \lambda^{2}} \int_{\mathbb{R}^{3}}\left\langle\varrho W(h, a) W(h, b)^{*} W\left(k, \lambda^{-2} x\right) \varphi_{\lambda},\right. \\
& \left.W\left(k, \lambda^{-2} x\right) \varphi_{\lambda}\right\rangle \sigma(d a, d b, d h)
\end{aligned}
$$

with

$$
\begin{aligned}
\left(R_{0} P_{\lambda} \varrho\right)(k, x)= & \frac{1}{2 \pi \lambda^{2}} \sum_{n=1}^{\infty} \int_{\mathbb{R}}\left\langle\varrho W\left(k, \lambda^{-2} x\right) \varphi_{\lambda},\right. \\
& \left.W\left(k, \lambda^{-2} x\right) \varphi_{\lambda}\right\rangle\left|\hat{\mu}_{n}(k-h / 2)\right|^{2} v_{n}(d h)
\end{aligned}
$$


The quantity to estimate is

$$
\begin{aligned}
& \int_{\mathbb{R}}\left|\left(P_{\lambda}(\varrho R)\right)(k, x)-\left(R_{0} P_{\lambda} \varrho\right)(k, x)\right| d k d x \\
& \leqq \frac{1}{2 \pi \lambda^{2}} \sum_{n=1}^{\infty} \int_{\mathbb{R}^{5}} \mid\left\langle\varrho W(h, a) W(-h,-b) W\left(k, \lambda^{-2} x\right) \varphi_{\lambda}, W\left(k, \lambda^{-2} x\right) \varphi_{\lambda}\right\rangle \\
& \quad-\left\langle\varrho W\left(k, \lambda^{-2} x\right) \varphi_{\lambda}, W\left(k, \lambda^{-2} x\right) \varphi_{\lambda}\right\rangle \\
& \quad \cdot \exp [i(k-h / 2)(b-a)]|| \mu_{n}|(d a)| \mu_{n} \mid(d b) v_{n}(d h) d k d x .
\end{aligned}
$$

By the dominated convergence theorem it is sufficient to prove that for every $a, b, h$ the following quantity converges to zero with $\lambda$.

$$
\begin{aligned}
J_{\lambda}= & \frac{1}{2 \pi} \int_{\mathbb{R}^{2}} \mid\left\langle\varrho W(h, a) W(-h,-b) W(k, x) \varphi_{\lambda}, W(k, x) \varphi_{\lambda}\right\rangle \\
& -\left\langle\varrho W(k, x) \varphi_{\lambda}, W(k, x) \varphi_{\lambda}\right\rangle \exp [i(k-h / 2)(b-a)] \mid d k d x \\
= & \frac{1}{2 \pi} \int_{\mathbb{R}^{2}} \mid\left\langle\varrho W(0, a-b) W(k, x) \varphi_{\lambda}, W(k, x) \varphi_{\lambda}\right\rangle \\
& -\left\langle\varrho W(k, x) \varphi_{\lambda}, W(k, x) \varphi_{\lambda}\right\rangle \exp [i k(b-a)] \mid d k d x \\
= & \frac{1}{2 \pi} \int_{\mathbb{R}^{2}} \mid\left\langle\varrho W(k, x) W(0, a-b) \varphi_{\lambda}, W(k, x) \varphi_{\lambda}\right\rangle \\
& -\left\langle\varrho W(k, x) \varphi_{\lambda}, W(k, x) \varphi_{\lambda}\right\rangle \mid d k d x \\
= & \left\|P_{W(0, a-b) \varphi_{\lambda} \cdot \varphi_{\lambda}}^{\lambda} \varrho-P_{\varphi_{\lambda} \cdot \varphi_{\lambda} \varrho} \varrho\right\|_{1} \\
\leqq & \left\|W(0, a-b) \varphi_{\lambda}-\varphi_{\lambda}\right\|\left\|_{\varrho}\right\|_{\operatorname{tr}}
\end{aligned}
$$

which converges to zero as $\lambda \rightarrow 0$.

- By taking adjoints we similarly find that

$$
\int_{\mathbb{R}^{2}}\left|\left(P_{\lambda}(R \varrho)\right)(k, x)-\left(R_{0} P_{\lambda} \varrho\right)(k, x)\right| d k d x
$$

converges to zero with $\lambda$ in a suitable manner.

Note Added in Proof. A proof of Theorem 2.1 may also be found in Kato, T.: On a matrix limit theorem. Linear Multilinear Algebra 3, 67-71 (1975)

\section{References}

1. Davies, E. B.: Quantum stochastic processes. Commun. math. Phys. 15, 277-304 (1969)

2. Davies, E. B.: Quantum stochastic processes II. Commun. math. Phys. 19, 83-105 (1970)

3. Davies, E. B.: Some contraction semigroups in quantum probability. Z. Wahrscheinlichkeitsth. 23, 261-273 (1972)

4. Davies, E. B.: Markovian master equations. Commun. math. Phys. 39, 91-110 (1974)

5. Davies, E. B.: Markovian master equations II. Math. Ann. 219, 147-158 (1976)

6. Dynkin,E. B.: Markov processes, Vol. 1. Berlin-Heidelberg-New York: Springer 1961

7. Ellis, R.S., Pinsky, M.A.: Asymptotic nonuniqueness of the Navier-Stokes equation in kinetic theory. Bull. Amer. Math. Soc. 80, 1160-1164 (1974)

8. Gorini, V., Kossakowski, A.: $N$-level system in contact with a singular reservoir. Preprint (1975) 
9. Haake, F.: Statistical treatment of open systems by generalized master equations. Springer tracts in modern physics 66. Berlin-Heidelberg-New York: Springer 1973

10. Hepp, K.: The classical limit for quantum-mechanical correlation functions. Commun. math. Phys. 35, 265-277 (1974)

11. Kossakowski,A.: On quantum statistical mechanics of nonhamiltonian systems. Rep. Math. Phys. 3, 247-274 (1972)

12. Lindblad, G.: On the generators of quantum dynamical semigroups. Preprint (1975)

13. Martin, P., Emch, G.: A rigorous model sustaining Van Hove's phenomenon. Helv. Phys. Acta 48, 59-78 (1975)

14. Papanicolaou, G.C., Kohler, W.: Asymptotic analysis of deterministic and stochastic equations with rapidly varying components. Commun. math. Phys. 45, 217-232 (1975)

15. Pulè, J.: The Bloch equations. Commun. math. Phys. 38, 241-256 (1974)

Communicated by K. Hepp

Received February 27, 1976 
